\title{
Intraoperative mapping of the cortical areas involved in multiplication and subtraction: an electrostimulation study in a patient with a left parietal glioma
}

\author{
H Duffau, D Denvil, M Lopes, F Gasparini, L Cohen, L Capelle, R van Effenterre
}

J Neurol Neurosurg Psychiatry 2002;73:733-738

See end of article for authors' affiliations

Correspondence to: Dr H Duffau, Service de Neurochirurgie, Hôpital de la Salpêtrière, $47 \mathrm{Bd}$ de l'hôpital, 75651 Paris,

Cedex 13, France;

hugues.duffau@

psl.ap-hop-paris.fr

Received

19 February 2002

In final revised form 24 July

2002

Accepted 8 August 2002

\begin{abstract}
Objectives: Advances in neuroimaging studies have recently improved the understanding of the functional anatomy of the calculation processes, having in particular underlined the central role of the angular gyrus (AG). In this study, the authors applied this knowledge to the surgical resection of a glioma invading the left $A G$, by localising and sparing the cortical areas involved in two different components of calculation (multiplication and subtraction), using direct electrical stimulations.

Methods: A calculation mapping was performed in a patient without deficit except a slightly impaired performance for serial arithmetic subtraction, during the resection under local anaesthesia of a left parieto-occipital glioma invading the dominant AG. After somatosensory and language mappings, cortical areas involved in single digit multiplications and subtractions of seven were mapped using the method of electrostimulation, before glioma removal.

Results: Distinct sites specifically involved in multiplication or subtraction were detected within the left $A G$, with a precise spatial distribution and overlapping. All the eloquent (somatosensory, language, and calculation) areas were surgically spared. Postoperatively, the patient had a transient complete deficit for arithmetic subtraction, without either multiplication or language disturbance. The tumour removal was complete.

Conclusions: These findings suggest: firstly, the usefulness of an intraoperative calculation mapping during the removal of a lesion involving the left dominant $A G$, to avoid permanent postoperative deficit of arithmetic processes while optimising the quality of tumour resection; secondly, the possible existence of a well ordered and dynamic anatomo-functional organisation for different components of calculation within the left AG.
\end{abstract}

$\mathrm{E}$ ver since Henschen's seminal study, ${ }^{1}$ evidence from neuropsychological reports in brain damaged patients $s^{2-8}$ and functional neuroimaging studies ${ }^{9-21}$ points toward a major involvement of inferior parietal regions in mental calculation. Moreover, there are indications that these areas include arithmetic components that may be selectively impaired or preserved following lesions.. ${ }^{2} 8$ 17 22-26 Indeed, parietal damage can induce dissociated disorders of various types of calculation. ${ }^{2378}$ In the same way, acalculia may be isolated ${ }^{8}$ or associated with impairment of other cognitive functions, ${ }^{27}$ such as aphasia or Gerstmann's syndrome.

Development of positron emission tomography and functional MRI, have recently improved the knowledge of the distributed networks that subserve calculation abilities, including prefrontal, premotor, and parietal cortices. ${ }^{1113} 141_{17-2128}^{29} \mathrm{In}$ particular, a clear cut distinction is emerging between the left angular gyrus (AG) proper, showing more activation for language dependent calculation (multiplication and exact calculation), and a more anterior bilateral intraparietal site, showing greater activation during non-rote quantity manipulations (such as subtraction and approximate calculation). ${ }^{11} 1720$

In this report, we applied this knowledge to the surgical resection of a glioma invading the left AG, by performing under local anaesthesia an intraoperative electrostimulation mapping of the cortical areas involved in calculation processes. Our goals were first to avoid permanent postoperative calculation deficit, and to study the involvement of different left parietal regions in multiplication versus subtraction, two operations that can be doubly dissociated after parietal lesions. On the basis of these findings, the anatomo-functional organisation of the AG, its integration in calculation networks, and its interaction with language and working memory functions are discussed.

\section{METHODS}

Case presentation

Patient

The patient was a 44 year old right handed nurse. She was operated on in our institution for a left parieto-occipital low grade glioma that was causing partial seizures.

The preoperative clinical examination, performed by a neurologist, was normal. There was no visuospatial deficit. Language was unimpaired including spontaneous speech, word generation, repetition, picture naming, writing, and reading.

Assessment of calculating skills revealed that the patient was able to read Arabic numerals aloud, to write Arabic numerals to dictation, to compare pairs of single word and multi-words numerals, and to recite multiplication tables without any error. However, although written complex calculations (multi-digit addition, multiplication, subtraction, and division problems) were normal (100\% of good answers), mental serial subtraction by seven was slightly impaired in comparison with the performance of the age matched controls (20\% of errors on 10 items-that is, only $80 \%$ of good answers). Moreover, the number span was restricted to four items forward and two in the backward order.

Handedness was assessed using a standardised questionnaire. ${ }^{30}$ Hemispheric dominance for language was assessed with preoperative functional MRI using semantic fluency, covert sentence repetition, and story listening tasks. ${ }^{31}$

The preoperative anatomical MRI showed a left tumour located at the parieto-occipital junction, involving the superior 

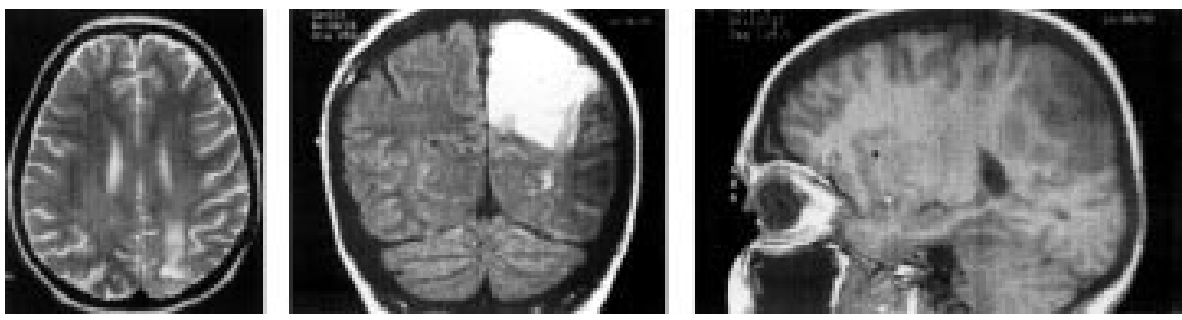

Figure 1 Preoperative T2 weighted axial, FLAIR weighted coronal, and T1 weighted enhanced MRI, showing a left glioma at the parieto-occipital junction, involving the superior parietal lobule, and the postero-superior part of the inferior parietal lobule.

parietal lobule, and the postero-superior part of the angular gyrus (fig 1).

\section{Intraoperative electrostimulation technique}

Because of the location of the tumour in the dominant hemisphere, it was decided to perform awake, intraoperative functional mapping, before and during surgical resection, to minimise the risk of neurological sequelae. ${ }^{3233}$ The patient gave her written informed consent.

The cortico-subcortical map was obtained using the method of electrostimulation previously described by the authors. ${ }^{34}{ }^{35}$ Briefly, a $5 \mathrm{~mm}$ spaced tips bipolar probe delivering a biphasic current was applied during four seconds on the brain (pulse frequency of $60 \mathrm{~Hz}$, single pulse phase duration of $1 \mathrm{~ms}$, amplitude of $6 \mathrm{~mA}$, Ojemann cortical stimulator, Radionics, Burlington, MA). Mapping under local anaesthesia was first performed in the central region, to identify the primary somatosensory areas (eliciting paraesthesias in the controlateral hemibody when stimulated). Then, we detected sites where stimulation induced speech arrest during self paced verbal counting (regurlarly in order from 1 to 10 , and so on), or anomia or paraphasias during picture naming.

\section{Experimental calculation tasks}

Preoperative MRI and intraoperative ultrasonography showed that the tumour was located at the parieto-occipital junction with infiltration of the postero-superior part of the AG. Therefore, the areas involved in mental calculation were also mapped during surgery.

Because of time constraints during the awake surgical procedure, all the stimuli were only presented verbally and not also visually using Arabic digits. For the same reason, only multiplication and subtraction were studied, and not addition and division.

Thus, the patient was asked to perform two different calculation tasks:

(1) Simple multiplication problems, for example, single digit multiplications systematically with one operand $<6$ (for instance $4 \times 4 ; 3 \times 7 ; 2 \times 6 ; 5 \times 8 \ldots$. . . No complex multidigit multiplication was performed, essentially because of the fact that the electrical stimulation should not exceed four seconds to avoid intraoperative seizures-thus, necessitating that all the operations were presented and solved by the patient within this delay.

(2) Subtraction of seven from one or two digit numbers (for example, 96-7, then 9-7 ...).

Multiplication and subtraction problems were alternatedthree multiplications then three subtractions, and so on. During one "calculation block" (for instance three multiplications), one task was performed without stimulation, a second one performed during electrostimulation, and the third one again without any stimulation (to check if the patient was again able to solve the problem, especially when the previous stimulation had induced a disruption of calculation). The same problems were presented for both stimulated and non-stimulated trials, but never consecutively. For instance:

- one "multiplication block": $4 \times 4$ (without stimulation), then $3 \times 7$ (with stimulation), then $2 \times 6$ (without stimulation);
- then one "subtraction block": 96-7 (without stimulation), then 9-7 (with stimulation), then 54-7 (without stimulation);

- again one "multiplication block": $5 \times 8$ (without stimulation), then $4 \times 4$ (with stimulation), then $3 \times 7$ (without stimulation);

- again one "subtraction block": 80-7 (without stimulation), then 96-7 (with stimulation), then 9-7 (without stimulation);

- and so on.

The patient was never informed when the brain was stimulated. The same cortical site was never stimulated successively twice, to avoid seizures. At each stimulation site, the patient performed three blocks of subtraction and three blocks of multiplication, for a total of three stimulated problems and six non-stimulated problems for each type of operation. The whole left parietal lobe exposed by the boneflap was mapped for calculation (supramarginalis gyrus, AG-both parts involved and non-involved by the glioma-, superior parietal lobule), except the areas previously demonstrated during the counting task as inducing speech arrest when stimulated.

The tumour removal was conducted using these functional landmarks as boundaries of the resection, at the cortical as well as at the subcortical level, as stimulations were continuously performed all along the surgery to identify the eloquent pathways.

\section{Postoperative course}

At three days and seven days after the surgery, the patient underwent the same neurological examination as preoperatively.

In the numerical domain, she was asked:

- to read Arabic numerals aloud,

- to write Arabic numerals to dictation,

- to compare pairs of single word and multi-words numerals,

- to solve orally presented single digit multiplication problems mentally,

- to solve orally presented single digit and multi-digit subtraction problems mentally.

MRI was performed three hours after the surgical procedure to evaluate the quality of glioma resection.

\section{RESULTS}

\section{Intraoperative mapping}

The tumour was first delineated using ultrasonography, and boundaries marked by letter tags on the brain surface. The functional stimulation mapping then permitted identification of the following cortical sites (fig 2):

- primary somatosensory areas of the hand and fingers (tags $10,11,12$ ), located within the retrocentral gyrus, in front of the intraparietal sulcus;

- a site located in the inferior part of the supramarginalis gyrus, where stimulations induced complete speech arrest $(\operatorname{tag} 20)$; 


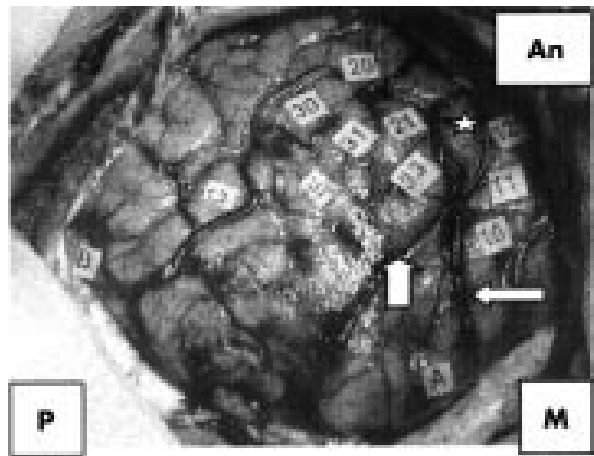

Figure 2 Intraoperative view before resection. The letters marked the tumour boundaries. The number tags show the eloquent areas identified using electrical stimulations: primary somatosensory areas of the hand and fingers $(10,11,12)$; language sites $(20$ : counting, 21: naming); calculation areas (30: multiplication, 31 : multiplication and subtraction, 33: subtraction). An: anterior; P: posterior; $M$ : midline; small arrow: retrocentral sulcus; large arrow: intraparietal sulcus, star: supramarginalis gyrus.

- a site within the superior part of the supramarginalis gyrus, located above the previous site, where stimulation interfered with naming ( $\operatorname{tag} 21)$;

- three areas involved in calculation, all located within the anterior part of the angularis gyrus, immediately behind the language sites and in front of the tumour. From bottom to top, was identified a site with a selective transient disruption of multiplication during stimulation (tag 30$)$, a site with a disruption of both multiplication and subtraction ( $\operatorname{tag} 31)$, and a site with a disruption of subtraction only ( $\operatorname{tag} 33$ ).

Whenever stimulation interfered with calculation, the patient did not produce an erroneous response but said "I don't know". Actually, the patient was instructed to say "I don't know" whenever she did not find the solution of the problem. This procedure allowed us to confirm that the calculation deficit did not reflect a non-specific speech arrest (for instance a seizure attributable to stimulation).

Thus, there was $100 \%$ of acalculia specifically for multiplication induced during three stimulations at the level of tag 30 (for example, no answer except "I don't know") - in comparison with $100 \%$ of good answers during six multiplications performed without any stimulations. There was also $100 \%$ of good answers during nine subtractions (three with stimulation, six without stimulation).

There was $100 \%$ of acalculia specifically for subtraction during three stimulations at the level of tag 33-in comparison with $100 \%$ of good answers during six subtractions performed without any stimulations. There was also $100 \%$ of good answers during nine multiplications (three with stimulation, six without stimulation).

There was $100 \%$ of acalculia for both operations generated during six stimulations (three during multiplication, three during subtraction) at the level of tag 31 -in comparison with $100 \%$ of good answers during 12 calculation trials (six multiplications, six subtractions) performed without any stimulations.

No other functional site was detected elsewhere on the brain surface exposed, in particular no eloquent area within the tumour.

There was no intraoperative seizure.

During removal of the lesion, a subcortical pathway eliciting anomia when stimulated was identified deeply (tag 40), at the level of the lateral wall of the ventricle (which was surgically opened). The resection was then stopped according to the functional cortico-subcortical boundaries provided by electrostimulation, namely with removal of the entire superior parietal lobule, the posterior part of the inferior lobule, and the

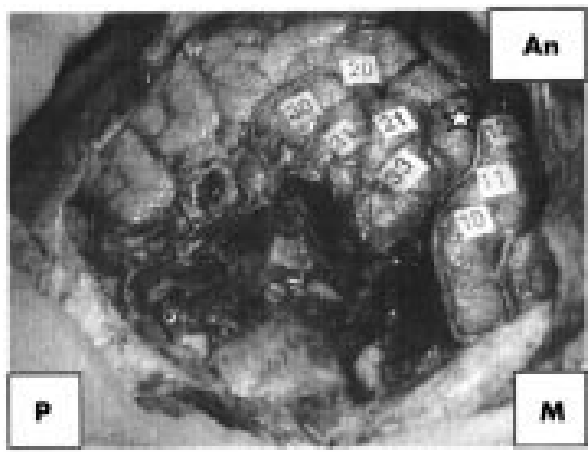

Figure 3 Intraoperative view after glioma removal. The same functional sites inducing the same responses during stimulations than before resection were identified all along the surgical procedure.

Moreover, a subcortical pathway eliciting anomia when stimulated was identified deeply (40) at the level of the lateral wall of the ventricle (infero-anterior wall of the surgical cavity). An: anterior; P: posterior; $M$ : midline, star: supramarginalis gyrus.

anterior part of the occipital lobe that was invaded by the glioma (fig 3). White matter stimulation never induced any disruption of calculation.

\section{Postoperative course}

Three days after surgery, the patient had no motor or language disturbance (spontaneous speech, word generation, repetition, and naming were normal). The examination of sensory functions showed a slight impairment of proprioception in the right upper limb, and a right inferior quadrantanopia, hampering a precise visual guided reaching.

In the numerical domain, the patient was able to read and write Arabic numerals, and to compare pairs of single word and multi-words numerals without error. Single digit multiplication problems were solved without error but abnormally slowly (error rate, $0 / 10$ ). Mental arithmetic subtraction was completely impossible, but in $50 \%$ of trials, the results were those of the corresponding addition (for example, $10-7=17$ or even $4-2=6$ ) ( $100 \%$ of error, 10 of 10$)$, may be related to problems of attention.

Moreover, the numeral span was reduced to two items (impossible in the reversed order).

At this time, the patient was too tired to undergo further testing.

Seven days after the surgical procedure, the somatic examination was normalised, except for the right inferior quadrantanopia. Before discharge, calculation tasks were performed . The patient was again able to read and write arabic numerals, and to compare pairs of single word and multi-words numerals without error and more quickly than before. Moreover, single digit multiplication problems were solved more easily and faster than four days before, and without any error (error rate, 0 of 20). Subtraction problems were solved without any error (even serial subtractions like 100-7-7 ...), except for the more complex multi-digit subtraction (for example, 47-13) with still an error rate of $40 \%$.

Finally, the numeral span improved (four items forward and four in the reversed order, as preoperatively).

The immediate postoperative MRI showed a complete tumour removal, and confirmed the resection of the entire superior parietal lobule, the posterior part of the inferior parietal lobule, and the parieto-occipital junction (fig 4).

\section{DISCUSSION}

This report of an intraoperative calculation mapping during resection of a left parieto-occipital glioma underlines two points of interest: surgical and pathophysiological. 

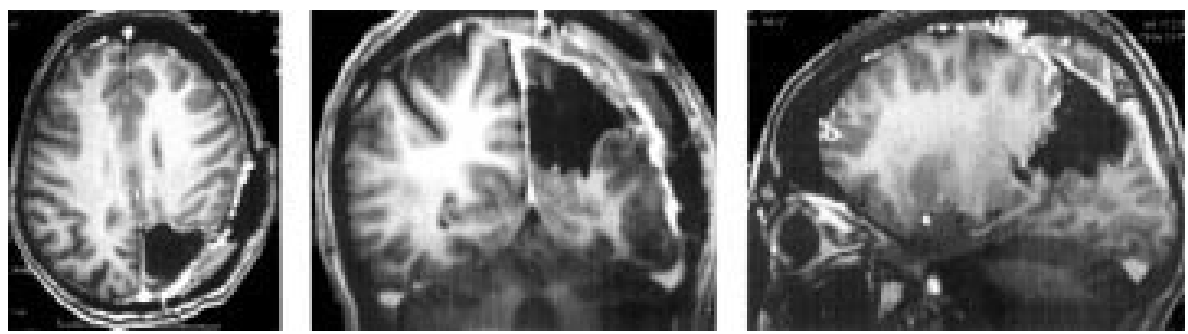

Figure 4 Postoperative axial, coronal, and sagittal Tl weighted enhanced MRI three hours after surgery, showing a total tumour removal, with resection of the entire superior parietal lobule, the posterior part of the inferior parietal lobule, and the parieto-occipital junction.

\section{Surgical interest}

Most recent series show that the regular use of the method of intraoperative brain electrostimulation during surgery in eloquent areas improved postoperative functional results. ${ }^{3436-38}$ Currently, direct stimulations are a safe, precise, and reliable method to identify the cortical and subcortical eloquent areas indispensable for sensorimotor, and even cognitive functions in awake patients. ${ }^{32} 339$ Indeed, it was demonstrated for cognitive functions, particularly language, that stimulations induce a transient inhibition of the function when applied on the essential cerebral structures-both grey or white matter. ${ }^{39}{ }^{40}$ However, very few attempts were made to map specifically calculation areas during surgery of lesion involving the left dominant parietal lobules, as only Whalen reported such an observation, testing only multiplication. ${ }^{41}$

In this study, we showed that calculation processes involved in multiplication and subtraction could be transitorily disrupted by direct cortical electrostimulation, permitting identification and sparing, during tumour removal, cortices essential for calculation. The results of intraoperative mapping were confirmed by the postoperative clinical course, as even though the patient had a transient deficit in subtraction (for which the cortical sites were the nearest to the surgical cavity), there was a recovery, proving that the all crucial calculation sites were preserved. Thus, the removal of an infiltrative glioma (to improve the quality of resection while minimising the risk of sequelae) can be considered using functional boundaries-represented not only by sensorimotor and language sites as previously described, ${ }^{32}{ }^{36-38}$ but also by calculation areas.

Nevertheless, these preliminary findings need to be confirmed with larger number of patients, and extended by a more extensive intraoperative mapping of a wider variety of arithmetic taks (that is, with incorporation of at least addition and division tasks) - with the aim to avoid any permanent postoperative calculation deficit after surgery of lesions involving the left parietal lobules (in particular the AG).

\section{Pathophysiological interest}

Calculation and the parietal lobe: the central role of the angular gyrus

Our intraoperative results clearly show that all the cortical sites whose stimulations elicited transient disruption of different forms of calculation were located exclusively within the AG. This finding supports the theory of Peritz and Henschen, who suggested that the left AG harboured a specific "calculation centre". ${ }^{42}$ Although this idea is not a new one, the use of electrostimulation has permitted confirmation of this hypothesis by a direct precise and reliable method. Indeed, deficits in calculation have been associated with a great variety of lesion locations in patients, and an overview of lesion studies led merely to the conclusions that left posterior cortical damage is more likely than anterior damage to affect mental calculation. ${ }^{43}{ }^{44}$ The difficulty in demonstrating that the AG constitutes the functional (parietal) epicentre for number processing in brain damaged patient can be partly explained by the fact that many lesions involved several parietal structures (superior lobule, postcentral gyrus, supramarginalis gyrus, AG). ${ }^{2}$

More recently, the development of functional neuroimaging methods improved the knowledge of distributed networks that subserve the performance of calculation tasks: activations during calculation tasks were tightly concentrated to the AG and the intraparietal sulcus.9 ${ }^{11} 1720$ However, these noninvasive techniques do not permit clear differentiation of the cortical areas involved in calculation, from those really essential for this function. Consequently, electrostimulation data seem to represent a substantial complement to the knowledge of the organisation of arithmetical areas, as the method evaluates the effect of a transient, limited, "evolving" and not "fixed" lesion in the same patient performing alternately different types of calculation tasks. This permits identification of the eloquent sites indispensable for the function.

Therefore, even taking into account the likely existence of an individual anatomo-functional variability, ${ }^{19} 4546$ these results give further support in favour of a key role of the left AG in mental calculation. It is also noted that the intraoperative data have been supported by the postoperative course, namely the recovery of arithmetic processing despite a total resection of the superior parietal lobule.

\section{Angular gyrus organisation: distribution of the distinct multiplication/subtraction areas}

Our study also argues in favour of the existence of distinct calculation sites within the AG: a site specifically involved in rote multiplication, and a site specifically involved in subtraction-plus an intermediary area (overlapping of the two previous sites) involved in both processings.

Indeed, the use of intraoperative electrical stimulations permitted performance of an accurate mapping $(5 \mathrm{~mm}$ of resolution) of the AG during two calculation tasks: multiplication and subtraction. Our results showed the existence of distinct anatomical sites specifically inhibited by stimulation during either multiplication or substraction; a multiplication area in the inferior part of the AG, and subtraction area in the superior part immediately below the intraparietal sulcus and behind the supramarginalis gyrus. There was also an overlapping site for both functions ( $\operatorname{tag} 31$ ). Such an organisation was confirmed by the postoperative course, namely a transient but complete specific subtraction deficit (while the multiplication was slow but possible), after resection that came very close to the superior part of the angular gyrus. The patient also presented with $20 \%$ of errors during subtraction tasks, corresponding to a level of performance considered to be impaired compared with that of her age matched controls (probably because of the immediate vinicity of the corresponding cortical site with the tumour).

Interestingly, this distribution seems very similar to the somatotopy widely described within the primary motor area-that is, distinct representations for each movement ${ }^{47}$ but with overlapping permitting a dynamic organisation, ${ }^{48}$ corresponding to parallel networks that enable neuronal recruitment for complex motor sequences, new skill acquisitions, ${ }^{49}$ or functional recovery after damage. ${ }^{45}$ 
Consequently, we hypothesise the existence within the angular gyrus of a possible dynamic "calculotopy", based on a well ordered distribution of specific calculation sites, nevertheless with overlapping.

Obviously, as a low grade glioma tends to be a slowly growing tumour, it cannot be certain that the functional re-organisation did not take place for calculation on this patient, as previously described for sensorimotor and language functions. ${ }^{40}{ }^{45}{ }^{46}$ However, our results seem in concordance with existing data from the literature: Cohen et al have described the case of a patient with multiplication problems despite subtraction preservation. The lesion affected the parietal perisylvian cortex, while a subset of the left inferior parietal lobule close to the intraparietal sulcus was spared ${ }^{2}$; interestingly, this area could correspond to the superior part of the AG suggested to represent the "subtraction cortex" in our patient (tag 33). Other authors also reported cases of patients who suffered from an intracranial haemorrhage at the left parieto-temporal junction, again with preservation of the superior part of the left inferior parietal cortex (near the intraparietal sulcus), with impairment in multiplication but not in subtraction. ${ }^{17} 24$

Secondly, a previous intraoperative calculation mapping study showed that electrostimulation of the left parietal lobe induced a transient deficit of the arithmetic processes. ${ }^{41}$ Although only mutiplication was studied in this report, with no attempt made to dissociate the areas involved in the different components of mental calculation, it is interesting to note that the multiplication disturbances were induced during stimulation of the inferior parietal cortex located immediately above and behind the end of the sylvian fissure, ${ }^{41}$ that is, very close to the "multiplication area" detected in our work (tag 30).

Thirdly, recent neuroimaging studies argued in favour of a dissociation between the left AG proper, activated in arithmetic fact (for example, in multiplication more than in subtraction ${ }^{11}{ }^{17}$ and in exact more than in approximate calculation ${ }^{20}$ ), and a more anterior and intraparietal site activated more during actual calculation such as subtraction (potentially associated to a prefrontal and/or right parietal activation ${ }^{2}$ 14-16 $1920^{20}$ ). Our results fit very well with these data, as the "subtraction area" identified by stimulation in the present work is more anterior and superior (immediately below the intraparietal sulcus and at the junction with the supramarginalis gyrus) than the "multiplication cortex" within the inferior part of the AG.

In conclusion, cortical areas involved in multiplication and subtraction seem to be distinct within the left AG, explaining that patients may be selectively impaired in one of these operations with preservation of the other one. ${ }^{23} 81722-26$

\section{CONCLUSIONS}

These findings suggest:

- firstly, the usefulness of an intraoperative calculation mapping during the removal of lesion involving the left dominant $A G$, to avoid postoperative definitive deficit of arithmetic processing while optimising the quality of tumour resection;

- secondly, the possible existence of a well ordered and dynamic anatomo-functional organisation for different components of calculation within the left AG.

However, we must underline the fact that the surgical and pathophysiological considerations reported in this work are based on a single patient. Consequently, further studies are mandatory to confirm these preliminary data, with:

- a larger number of patients;

- a greater number of intraoperative stimuli, testing more arithmetic processes such as addition and division;
- if possible, a correlation for each patient between the results of intraoperative electrical calculation mapping and those of preoperative and postoperative functional neuroimaging performed during the same tasks.

Moreover, transcranial magnetic stimulations could be used to simulated intraoperative electrical stimulation with normal control participants, to better understand the anatomofunctional organisation of calculation networks.

\section{Authors' affiliations}

H Duffau, D Denvil, M Lopes, F Gasparini, L Capelle, R van Effenterre, Department of Neurosurgery, Hôpital de la Salpêtrière, Paris, France

L Cohen, Department of Neurology, Hôpital de la Salpêtrière H Duffau, L Cohen, L Capelle, Department of IFR 49, Hôpital de la Salpêtrière

Competing interests: none declared.

\section{REFERENCES}

1 Henschen SE. Clinical and anatomical contributions on brain pathology. Arch Neurol 1919;13:226-249.

2 Cohen L, Dehaene S, Chochon F, et al. Language and calculation within the parietal lobe: a combined cognitive, anatomical and fMRI study. Neuropsychologia 2000;38:1426-40.

3 Dehaene S, Cohen L. Cerebral pathways for calculation : double dissociation between rote verbal and quantitative knowledge of arithmetic. Cortex 1997;33:219-50.

4 Gerstmann J. Syndrome of finger agnosia, disorientation for right and left, agraphia and acalculia. Arch Neurol Psychiatry 1940;44:43-74.

5 Martins IP, Ferreira J, Borges L. Acquired procedural dyscalculia associated to a left parietal lesion in a child. Neuropsychol Dev Cogn Sect C Child Neuropsychol 1999;5:265-73.

6 Mayer E, Martory MD, Pegna AJ, et al. A pure case of Gerstmann syndrome with a subangular lesion. Brain 1999:122:1 107-20.

7 Takayama Y, Sugishita M, Akiguchi I, et al. Isolated acalculia due to left parietal lesion. Arch Neurol 1994;51:286-91.

8 Warrington EK. The fractionation of arithmetical skills : a single case study. Q J Exp Psychol A 1982;34:31-51

9 Burbaud P, Camus O, Guehl D, et al. A functional magnetic functional imaging study of mental substraction in human subjects. Neurosci Lett 1999;273:195-9.

10 Burbaud P, Camus O, Guehl D, et al. Influence of cognitive strategies on the pattern of cortical activation during mental substraction. A functional imaging study in human subjects. Neurosci Lett 2000;287:76-80.

11 Chochon F, Cohen L, van de Moortele PF, et al. Cortical and subcortical circuits for mental arithmetic : functional neuroimaging of bilateral intraparietal, frontal, cingular and caudate contributions to various calculation tasks. J Cog Neurosci 1999:11:617-30.

12 Cowell SF, Egan GF, Code C, et al. The functional anatomy of simple calculation and number repetition : a parametric PET activation study. Neuroimage 2000;12:565-73.

13 Dehaene S, Tzourio N, Frak V, et al. Cerebral activations during number multiplication and comparison : a PET study. Neuropsychologia 1996;34:1097-106.

14 Fulbright RK, Molfese DL, Stevens AA, et al. Cerebral activation during multiplication : a functional MR imaging study of number processing. Am J Neuroradiol 2000;21:1048-54.

15 Hayashi N, Ishii K, Kitagaki H, et al. Regional differences in cerebral blood flow during recitation of the multiplication table and actual calculation: a positron emission tomography study. J Neurol Sci 2000;176:102-8.

16 Kazui $\mathbf{H}$, Kitagaki $\mathrm{H}$, Mori E. Cortical activation during retrieval of arithmetical facts and actual calculation: a functional magnetic resonance imaging study. Psychiatry Clin Neurosci 2000;54:479-85.

17 Lee KM. Cortical areas differentially involved in multiplication and subtraction: a functional magnetic resonance imaging study and correlation with a case of selective acalculia. Ann Neurol 2000;48:657-61.

18 Pesenti $M$, Thioux M, Seron X, et al. Neuroanatomical substrates of arabic number processing, numerical comparison, and simple addition: a PET study. J Cog Neurosci 2000;1 2:461-79.

19 Rueckert L, Lange N, Partiot A, et al. Visualizing cortical activation during mental calculation with functional MRI. Neuroimage 1996;3:97-103

20 Stanescu-Cosson R, Pinel P, van De Moortele PF, et al. Understanding dissociations in dyscalculia : a brain imaging study of the impact of number size on the cerebral networks for exact and approximate calculation. Brain 2000;1 23:2240-55.

21 Zago L, Pesenti M, Mellet E, et al. Neural correlates of simple and complex mental calculation. Neuroimage 2001;13:314-27.

22 Dagenbach $\mathbf{D}, \mathrm{McCloskey} M$. The organization of arithmetic facts in memory : evidence from a brain-damaged patient. Brain Cogn 1992;20:345-66. 
23 Delazer M, Benke T. Arithmetic facts without meaning. Cortex 1997;33:697-710.

24 Lampl Y, Eshel Y, Gilad R, et al. Selective acalculia with sparing of the subtraction process in a patient with left parietotemporal hemorrhage. Neurology 1994:44:1759-61

25 McNeil J, Warrington EK. A dissocation between addition and subtraction with written calculation. Neuropsychologia 1994 ;32:717-28

26 Pesenti M, Seron X, Van der Linden M. Selective impairment as evidence for mental organization of arithetical facts: BB, a case of preserved subtraction? Cortex 1994:30:661-71.

27 Collignon R, Leclerq C, Mahy J. Etudes de la séméiologie des troubles du calcul observés au cours des lésion corticales. Acta Neurol Belg 1977;77:257-75.

28 Dehaene S, Spelke E, Pinel P, et al. Sources of mathematical thinking: behavorial and brain-imaging evidence. Science 1999;284:970-4.

29 Rickard TC, Romero SG, Basso G, et al. The calculating brain: an fMR study. Neuropsychologia 2000;38:325-35.

30 Harris AJ. Harris test of lateral dominance. Manual of directions for administration and interpretation. New York: Psychological Corporation, 1947

31 Lehéricy S, Cohen L, Bazin B, et al. Functional MR evaluation of temporal and frontal language dominance compared with the Wada test. Neurology 2000;54:1625-33.

32 Berger MS Ojemann GA. Intraoperative brain mapping techniques in neuro-oncology. Stereotactic Funct Neurosurg 1992;58:153-61.

33 Ojemann GA, Ojemann JG, Lettich E, et al. Cortical language localization in left, dominant hemisphere. An electrical stimulation mapping investigation in 117 patients. J Neurosurg 1989;71:316-26.

34 Duffau H, Capelle L, Sichez JP, et al. Intraoperative direct electrical stimulations of the central nervous system : the Salpêtrière experience with 60 patients. Acta Neurochirurg (Wien) 1999;141:1157-67.

35 Duffau H, Sichez JP, Lehéricy S. Intraoperative unmasking of brain redundant motor sites during resection of a precentral angioma. Evidence using direct cortical stimulations. Ann Neurol 2000:47:132-35.

36 Danks RA, Aglio LS, Gugino LD, et al. Craniotomy under local anesthesia and monitored conscious sedation for the resection of tumors involving eloquent cortex. J Neurooncol 2000;49:131-9.
37 Meyer FB, Bates LM, Goerss SJ, et al. Awake craniotomy for aggressive resection of primary gliomas located in eloquent brain. Mayo Clin Proc $2001 ; 76: 677-87$

38 Taylor MD, Bernstein M. Awake craniotomy with brain mapping as a routine surgical approach to treating patients with supratentorial intraaxial tumors : a prospective trial of 200 cases. J Neurosurg intraaxial tumors:

39 Duffau H, Capelle L, Sichez N, et al. Intraoperative mapping of the subcortical language pathways using direct stimulations: an anatomo-functional study. Brain 2002;125:199-214.

40 Duffau $\mathbf{H}$, Bauchet $L$, Lehéricy $\mathrm{S}$, et al. Functional compensation of the left dominant insula for language. Neuroreport 2001; 12:2159-63.

41 Whalen J, McCloskey M, Lesser RP, et al. Localizing arithmetic processes in the brain : evidence from a transient deficit during cortical stimulation. J Cognitive Neurosci 1997:9:409-17.

42 Peritz G. Zur pathopsychologie des rechnens. Deutsch Z Nervenheilkd 1918;61:234-340.

43 Dahmen W, Hartje W, Büssing A, et al. Disorders of calculation in aphasic patients-spatial and verbal components. Neuropsychologia 1982;20:145-53

44 Grafman J, Passafiume D, Faglioni P, et al. Calculation disturbances in adults with focal hemispheric damage. Cortex 1982;18:37-50.

45 Duffau $\mathbf{H}$. Acute functional reorganisation of the human motor cortex during resection of central lesions: a study using intraoperative brain mapping. J Neurol Neurosurg Psychiatry 2001;70:506-13.

46 Duffau H, Denvil D, Capelle L. Long term reshaping of language, sensory, and motor maps after glioma resection: a new parameter to integrate in the surgical strategy. J Neurol Neurosurg Psychiatry 2002;73:511-16.

47 Penfield W, Bolchey E. Somatic motor and sensory representation in the cerebral cortex of man as studied by electrical stimulations. Brain 1937;60:389-443

48 Sanes JN, Donoghue JP, Edelman RR, et al. Shared neural substrates controlling hand movements in human motor cortex. Science 1995;268: 1775-7.

49 Karni A, Meyer G. The acquisition of silled motor performance: fast and slow experience-driven changes in primary motor cortex. Proc Natl Acad Sci USA 1998;95:861-8.

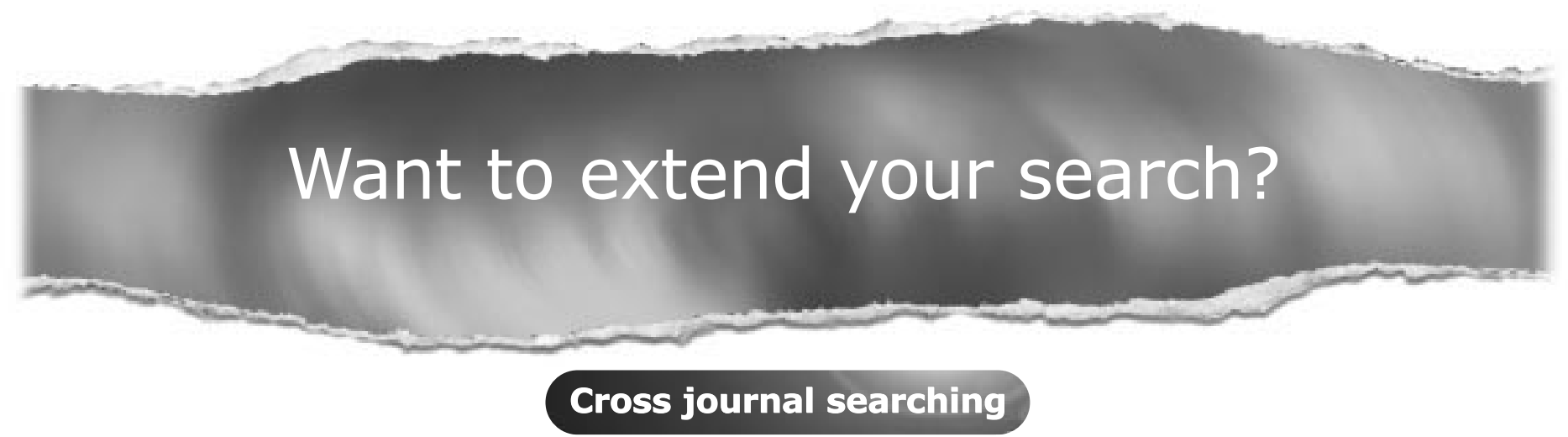

If you can't find what you are looking for in the Journal of Neurology, Neurosurgery, and Psychiatry you can extend your search across many of the more than 200 journals available for selection. You can restrict your search to specific subject areas (eg, clinical medicine, basic research), or select specific journals, or search all available titles.

\section{www.jnnp.com}

